\title{
Evaluation of the plate-rod model assumption of trabecular bone
}

\author{
Rodrigo Moreno, Magnus Borga and Örjan Smedby
}

\section{Linköping University Post Print}

N.B.: When citing this work, cite the original article.

(C2010 IEEE. Personal use of this material is permitted. However, permission to reprint/republish this material for advertising or promotional purposes or for creating new collective works for resale or redistribution to servers or lists, or to reuse any copyrighted component of this work in other works must be obtained from the IEEE.

Rodrigo Moreno, Magnus Borga and Örjan Smedby, Evaluation of the plate-rod model assumption of trabecular bone, IEEE International Symposium on Biomedical Imaging (ISBI) ,2012.

http://dx.doi.org/10.1109/ISBI.2012.6235586

Postprint available at: Linköping University Electronic Press

http://urn.kb.se/resolve?urn=urn:nbn:se:liu:diva-79480 


\title{
EVALUATION OF THE PLATE-ROD MODEL ASSUMPTION OF TRABECULAR BONE
}

\author{
Rodrigo Moreno $^{1,2} \quad$ Magnus Borga ${ }^{1,3} \quad$ Örjan Smedby ${ }^{1,2}$ \\ ${ }^{1}$ Center for Medical Image Science and Visualization (CMIV), Linköping University, Sweden \\ ${ }^{2}$ Department of Medical and Health Sciences (IMH), Linköping University, Sweden \\ ${ }^{3}$ Department of Biomedical Engineering (IMT), Linköping University, Sweden \\ Campus US, 581 85, Linköping, Sweden, \{rodrigo.moreno,magnus.borga,orjan.smedby\}@liu.se
}

\begin{abstract}
Trabecular bone has traditionally been assumed to be composed of plate- and rod-like trabeculae. This paper proposes a method to numerically evaluate the appropriateness of this assumption. In a first step, local constancy of thickness is estimated by comparing the maximum and mean diameter of the maximum inscribed balls centered at the medial axis/surface that includes every local point. In a second step, deviations from null curvature at the medial axis/surface are locally measured by comparing the geodesic and Euclidean distances from a point to its neighbors in the medial axis/surface. Finally, these two measurements are combined in order to locally estimate the compliance of the dataset with the plate-rod model assumption. Experiments on synthetic datasets show that the proposed measurements can be used to decide the compliance of a 3D shape with the plate-rod model. Results on micro computed tomography images show that the platerod model is more valid for a vertebra than for a radius. Thus, especially for the radius, measurements based on this model should be complemented with the proposed measurements.
\end{abstract}

Index Terms - Biomedical image analysis, trabecular bone, plate-rod model, shape classification, micro computed tomography

\section{INTRODUCTION}

Osteoporosis is a medical condition in which the strength of bones is reduced leading to an increased risk of fracture [1]. Research in trabecular bone has been fostered by evidence supporting that osteoporosis mainly affects the trabecular bone and that its architecture largely determines the biomechanical properties of the bone.

Trabecular bone has traditionally been assumed to be composed by a network of interconnected "plate-like" and "rod-like" trabeculae whose architecture varies with the skeletal site [2]. In this paper, this assumption will be referred to as the plate-rod model assumption. Based on this

This research has been supported by the Swedish Research Council (VR), grant no. 2006-5670. assumption, researchers have proposed methods for classifying trabecular bone, locally and/or globally, into plate-like and rod-like trabeculae and in order to independently estimate histomorphometric parameters for each type of trabeculae $[3,4,5,6,7,8]$. However, the plate-rod model should be seen as a simplification of the architecture of trabecular bone, since real trabecular bone is not composed of ideal plates and rods. Thus, estimations based on the plate-rod model assumption need to be complemented with an evaluation of the compliance of trabecular bone with this assumption. As an example, the structure model index [3, 4] is only valid for shapes that comply with the plate-rod model assumption, since the same value can be obtained for very different objects when it is applied to general shapes [9]. Despite this need, to the best of our knowledge, measurements to test the plate-rod model assumption have not been proposed so far.

Intuitively, plates and rods share two properties. Both have a constant thickness and the curvature at the medial axis/surface (MA for short) is null. Thus, the proposed measures in the next section consider deviations from these two properties to estimate the compliance of trabecular bone with the plate-rod model assumption.

The paper is organized as follows. Section 2 presents the proposed measures for testing the plate-rod model assumption on trabecular bone. Section 3 shows and discusses the results of experiments conducted on synthetic datasets and images acquired through Micro Computed Tomography $(\mu \mathrm{CT})$. Finally, Section 4 makes some concluding remarks.

\section{EVALUATION OF THE PLATE-ROD MODEL ASSUMPTION}

As already mentioned, both plates and rods are characterized by having a constant thickness and null curvature at the MA. Indeed, as shown in Figure 1, both conditions are necessary to comply with the plate-rod model assumption. Thus, the evaluation of this assumption can be divided into two steps: the independent evaluation of these two conditions in a first step and the combination of both evaluations into a single measurement in a second step. The following subsections de- 

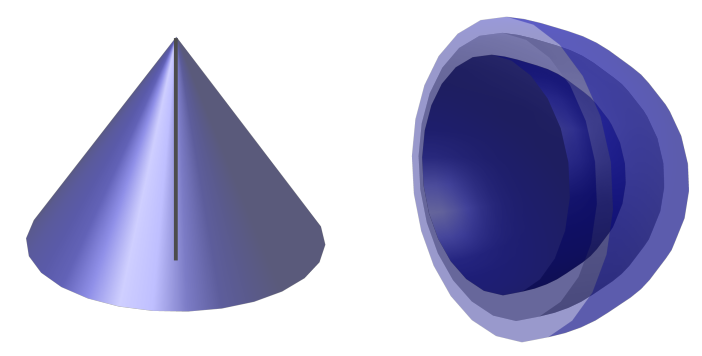

Fig. 1. Left: a cone has non-constant thickness and null curvature at the MA. Right: the difference between two concentric hemispheres has a constant thickness and non-null curvature at the MA. The MA is depicted in the middle of the two hemispheres.

scribe these steps.

\subsection{Local Constancy of Thickness}

The most widely used method for estimating thickness was proposed by Hildebrand and Rüegsegger [10]. The method computes local thickness at every point $x$ inside the trabecular bone as the diameter of largest inscribed ball centered at any point in the MA that includes $x$. More formally, let function $H(x)$ be defined as:

$$
H(x)=\left\{c \mid x \in B_{d(c), c}, c \in \operatorname{MA}(T)\right\},
$$

where $d(c)$ is twice the Euclidean distance transform computed at point $c, B_{d(c), c}$ is a ball of diameter $d(c)$ whose center is located at $c$ and $\mathrm{MA}(T)$ is the medial axis/surface of the trabecular bone $T$. Then, local thickness at $x, \operatorname{Th}(x)$ is estimated as [10]:

$$
\operatorname{Th}(x)=\max _{c \in H(x)} d(c) .
$$

It is important to remark that the cardinality of $H(x)$ is usually larger than one. Consequently, different statistics on the Euclidean distance of the points at $H(x)$ can be computed in order to be compared to the maximum. Thus, the proposed measure of local constancy of thickness takes advantage from the fact that the mean and the maximum only coincide at points where local thickness is constant. Let $T h_{m}(x)$ be twice the mean of the Euclidean distance transform of the points at $H(x)$, that is:

$$
T h_{m}(x)=\frac{1}{\operatorname{card}(H(x))} \sum_{c \in H(x)} d(c),
$$

where card refers to the cardinality of $H(x)$. Then, local constancy of thickness can be estimated through the new proposed $L$-measure, which is given by:

$$
L(x)=\frac{T h_{m}(x)}{\operatorname{Th}(x)} .
$$

The $L$-measure ranges from 0 to 1,1 representing a region with a completely constant thickness.

\subsection{Local Flatness/Straightness Estimation at the MA}

Flatness of surfaces and straightness of 3D curves can be defined as the degree to which a surface approximates a plane or a 3D curve approximates a straight line, respectively. Flatness or straightness can be estimated between two points, $c_{1}$ and $c_{2}$ belonging to the MA, by comparing the geodesic and Euclidean distance between them. This measure becomes either a flatness or a straightness estimate depending on whether both points lie on a surface or on a curve respectively. Thus, flatness or straightness between points $c_{1}$ and $c_{2}, \Gamma\left(c_{1}, c_{2}\right)$, can be estimated as:

$$
\Gamma\left(c_{1}, c_{2}\right)=\frac{d_{E}\left(c_{1}, c_{2}\right)}{d_{G}\left(c_{1}, c_{2}\right)},
$$

where $d_{G}$ and $d_{E}$ refer to geodesic and Euclidean distance respectively. Geodesic distances can be computed using a fast marching scheme [11] where the wave front movement is restricted to the MA, while computing Euclidean distances is trivial. Function $\Gamma$ takes values equal to or smaller than one for points lying on planes (straight lines) or curved surfaces (curves) respectively. There are two advantages of using $\Gamma$ for testing the null curvature at the MA instead of other curvature estimators. First, it makes unnecessary to determine the intrinsic local dimensionality at the MA, since it is appropriate both for flatness and straightness estimation. Second, it can be used in larger scales compared to other estimators such as the mean and Gaussian curvature [12].

Let $N\left(c_{1}\right)$ be a neighborhood at $c_{1}$. Local flatness or straightness at a point $c_{1}$ of the MA can be estimated by the new proposed $F S$-measure, which is given by:

$$
F S\left(c_{1}\right)=\frac{1}{\operatorname{card}\left(N\left(c_{1}\right)\right)} \sum_{c_{2} \in N\left(c_{1}\right)} \Gamma\left(c_{1}, c_{2}\right) .
$$

A relevant factor to consider in the estimation of the $F S$ measure is the scale. Taking into account that the scale is related to the local thickness, $N\left(c_{1}\right)$ has been set in the experiments of Section 3 to the points in a radius equivalent to the local thickness at $c_{1}$. In turn, the local flatness or straightness measure can also be extended to any point $x$ in the trabecular bone by computing the $F S$-measure as the mean of $F S$ measure for $c_{1} \in H(x)$, where $H(x)$ is the function defined in (1). Similarly to the $L$-measure, the $F S$-measure also ranges from 0 to 1,1 representing a flat region or a straight axis.

\subsection{Measure of Compliance with the Plate-Rod Model}

A measurement of compliance of a shape with the platerod model can be obtained through the new proposed $P R$ measure, which is computed as:

$$
P R(x)=L(x)^{\alpha} F S(x)^{\beta}
$$


Table 1. Mean and standard deviations (in parenthesis) of $L-$, $F S$ - and $P R$-measures respectively for the synthetic and $\mu \mathrm{CT}$ datasets, with $\alpha=4$ and $\beta=2$.

\begin{tabular}{lccc}
\hline Dataset & $L$ & $F S$ & $P R$ \\
\hline Rod & $0.99(0.00)$ & $0.99(0.00)$ & $0.98(0.04)$ \\
Plate & $1.00(0.00)$ & $1.00(0.00)$ & $0.99(0.01)$ \\
Synthetic 1 & $0.99(0.03)$ & $0.99(0.01)$ & $0.94(0.11)$ \\
Synthetic 2 & $0.97(0.07)$ & $0.99(0.01)$ & $0.91(0.20)$ \\
\hline Cone 1 & $0.86(0.07)$ & $0.99(0.00)$ & $0.52(0.19)$ \\
Cone 2 & $0.85(0.09)$ & $0.99(0.00)$ & $0.55(0.22)$ \\
Hemispheres 1 & $0.97(0.02)$ & $0.79(0.06)$ & $0.56(0.09)$ \\
Hemispheres 2 & $0.97(0.03)$ & $0.71(0.03)$ & $0.45(0.06)$ \\
\hline Radius & $0.93(0.06)$ & $0.92(0.05)$ & $0.65(0.18)$ \\
Vertebra & $0.95(0.07)$ & $0.96(0.03)$ & $0.76(0.24)$ \\
\hline
\end{tabular}

where $L$ and FS are the measures proposed in the previous subsections, and $\alpha$ and $\beta$ are parameters to weight the importance of local constancy of thickness and local flatness/straightness respectively.

\section{RESULTS AND DISCUSSION}

Experiments have been conducted on synthetic models and images of trabecular bone acquired through $\mu \mathrm{CT}$. For all the experiments, segmentation has been performed, and the MA computed as proposed in [13]. Table 1 shows the mean and the standard deviations of $L-, F S$ - and $P R$-measures for all tested datasets computed on the entire objects.

The first experiment was conducted on a single rod and a single plate. As expected, the $L$ - and $F S$-measures are close to one (see Table 1). The purpose of a second experiment on the synthetic models depicted in Figure 2(a-b), referred to as Synthetic 1 and 2 respectively in the table, was to assess the appropriateness of the new proposed measures for images that comply with the plate-rod model assumption. As expected, both, $L$ - and $F S$ - measures are close to one for these images (see Table 1), except for a small region around the intersections (see Figure 2). A third experiment was conducted to compute the measures for shapes that do not comply with the plate-rod model assumption, such as those depicted in Figure 1. In Table 1, Cone 1 and 2 refer to cones where the height is equal to the diameter and the radius of the base respectively. In turn, Hemispheres 1 and 2 refer to differences between concentric hemispheres where the thickness is once and twice the internal radius respectively. The $L$-measure is reduced to 0.85 for Cone 2, while the $F S$-measure is reduced to 0.71 for Hemispheres 2. These reductions can be used to tune parameters $\alpha$ and $\beta$. For example, assuming that Cone 2 and the Hemispheres 2 are extreme scenarios, $\alpha$ can be set to four and $\beta$ to two, so $P R$ will have a value close to 0.5 for these two cases. Although other strategies can be followed to tune these parameters, the aforementioned setting allows

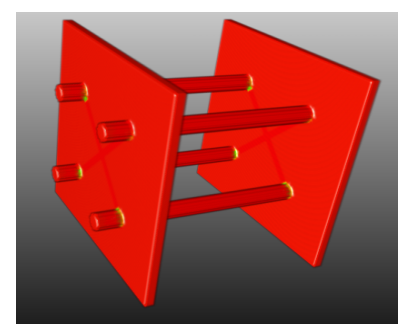

(a)

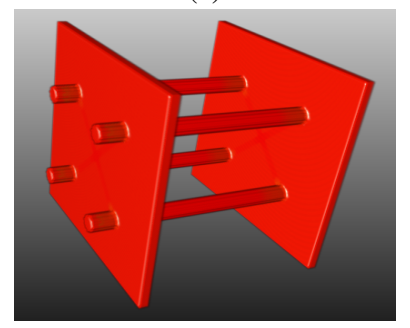

(c)

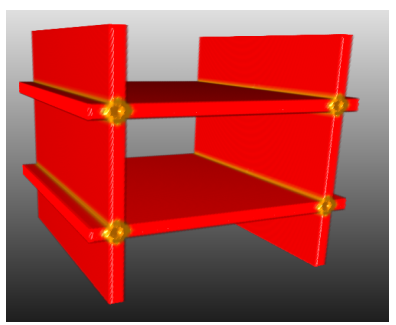

(b)

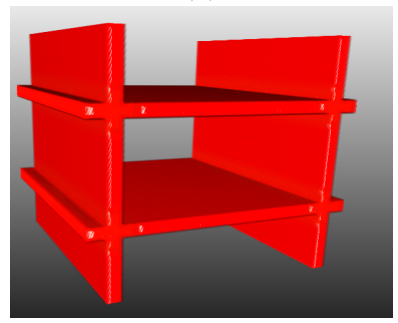

(d)
Fig. 2. (a-b): $L^{\alpha}$ of images Synthetic 1 and 2 respectively. (c-d): $F S^{\beta}$ of the same images. Red and green for the $L^{\alpha}$ - and $F S^{\beta}$-measures indicate values close and far from one respectively.

us to compare the $\mu \mathrm{CT}$ images with respect to these two extreme cases.

Figure 3 shows a rendering and the $L$ - and $F S$-measures with their respective histograms computed for two $\mu \mathrm{CT}$ images. These images correspond to volumes of interest of a vertebra and a radius respectively ${ }^{1}$. As seen on Figure 3 and Table 1, the vertebra better complies with the plate-rod model assumption than the radius since its trabeculae both have a more constant thickness and are more flat/straight. For example, the radius has large regions depicted in green for the $F S$ measure. Furthermore, Figures $3 \mathrm{~g}$ and $3 \mathrm{~h}$ show larger skewnesses of the histograms for the vertebra than for the radius, especially regarding the $F S$-measure. Although an extended validation is required, which is out of the scope of this paper, this preliminary result suggests that parameters based on the plate-rod model assumption could be more reliable when computed on vertebrae than on the radius. Also, the $P R$ measure of the radius is closer to the values of the Cone 2 and Hemispheres 2. That means that, especially for the radius, estimations based on the plate-rod model assumption need to be complemented with the proposed measurements.

\section{CONCLUDING REMARKS}

We propose a method to evaluate the plate-rod model assumption of trabecular bone based on measurements of the local constancy of thickness and the null curvature at the MA.

\footnotetext{
${ }^{1}$ We thank Prof. Osman Ratib from the Service of Nuclear Medicine of the Geneva University Hospitals for providing the $\mu \mathrm{CT}$ data of the vertebra and Andres Laib and Torkel Brismar for providing the $\mu \mathrm{CT}$ data of the radius.
} 


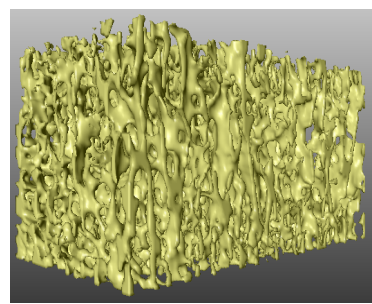

(a)

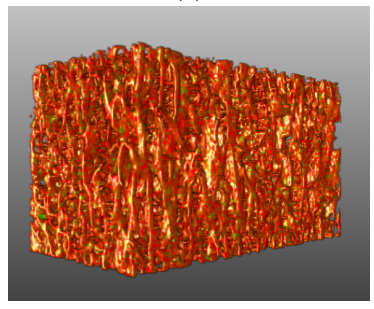

(c)

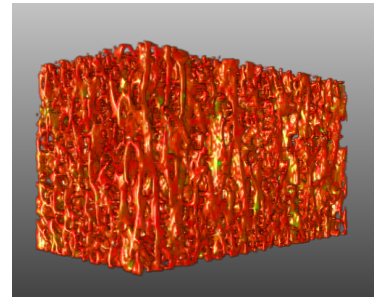

(e)

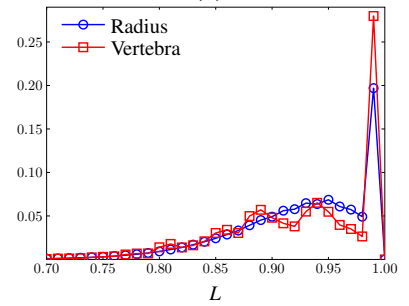

(g)

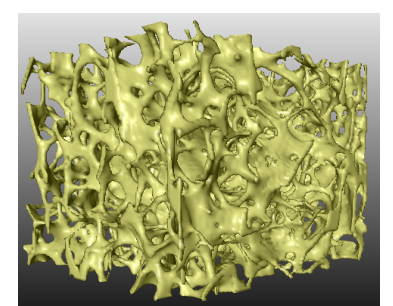

(b)

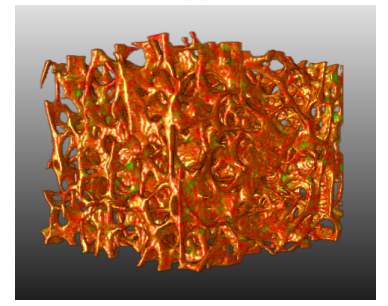

(d)

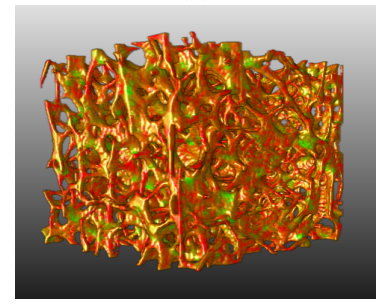

(f)

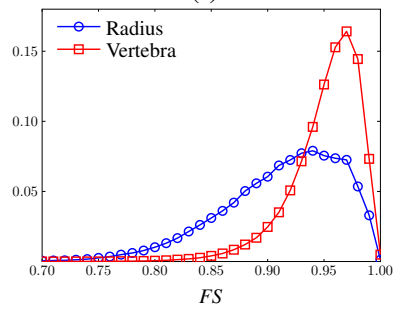

(h)
Fig. 3. (a-b): rendering of volumes of interest of a vertebra (left) and a radius (right). (c-d): $L^{\alpha}$. (e-f): $F S^{\beta}$. (g-h): histograms of $L$ - and $F S$-measures respectively for the vertebra and radius. The same color convention of Figure 2 has been used. The images have an isotropic resolution of $82 \mu \mathrm{m}$ and $20 \mu \mathrm{m}$ for the vertebra and radius respectively.

These two measurements are combined in order to locally estimate the compliance of the dataset with the plate-rod model assumption. Experiments on synthetic datasets show that the proposed measurement can be used to decide whether or not a shape complies with the plate-rod model. Results on micro computed tomography images show that the plate-rod model is more valid for the tested vertebra than for the tested radius. Thus, preliminary results suggest that, especially for the radius, measurements based on this model should be complemented with the proposed measurements. Plans for future research include testing trabecular bone from more skeletal sites, finding correlations between the proposed measurements and mechanical properties of the bone and computing these measurements in gray-scale in order to apply them to images acquired in vivo where segmentation is not trivial.

\section{REFERENCES}

[1] S. R. Cummings and L. J. Melton, "Epidemiology and outcomes of osteoporotic fractures," Lancet, vol. 359, no. 9319, pp. 1761-1767, 2002.

[2] T. Hildebrand, A. Laib, R. Müller, J. Dequeker, and P. Rüegsegger, "Direct three-dimensional morphometric analysis of human cancellous bone: microstructural data from spine, femur, iliac crest, and calcaneus," J. Bone Miner. Res., vol. 14, no. 7, pp. 1167-1174, 1999.

[3] T. Hildebrand and P. Rüegsegger, "Quantification of bone microarchitecture with the structure model index," Comput. Methods Biomech. Biomed. Eng., vol. 1, no. 1, pp. 15-23, 1997.

[4] Z. Tabor, "A novel method of estimating structure model index from gray-level images," Med. Eng. Phys., vol. 33, no. 2, pp. $218-225,2011$.

[5] P. Saha, Y. Xu, H. Duan, A. Heiner, and G. Liang, "Volumetric topological analysis: A novel approach for trabecular bone classification on the continuum between plates and rods," IEEE Trans. Med. Imaging, vol. 29, no. 11, pp. 1821 -1838, 2010.

[6] F. Peyrin, D. Attali, C. Chappard, and C. L. Benhamou, "Local plate/rod descriptors of 3D trabecular bone micro-CT images from medial axis topologic analysis," Med. Phys., vol. 37, no. 8, pp. 4364-4376, 2010.

[7] R. Moreno, Ö. Smedby, and M. Borga, "Soft classification of trabeculae in trabecular bone," in Proc. Int. Symp. Biomed. Imaging: From Nano to Macro (ISBI), 2011, pp. 1641 -1644.

[8] B. Vasilić, C. S. Rajapakse, and F. W. Wehrli, "Classification of trabeculae into three-dimensional rodlike and platelike structures via local inertial anisotropy," Med. Phys., vol. 36, no. 7, pp. 3280-3291, 2009.

[9] J. Ohser, C. Redenbach, and K. Schladitz, "Mesh free estimation of the structure model index," Image Anal. Stereol., vol. 28, no. 3, pp. 179-185, 2009.

[10] T. Hildebrand and P. Rüegsegger, "A new method for the model-independent assessment of thickness in threedimensional images," J. Microsc., vol. 185, no. 1, pp. 67-75, 1997.

[11] J. A. Sethian, Level Set Methods and Fast Marching Methods: Evolving Interfaces in Computational Geometry, Fluid Mechanics, Computer Vision and Materials Science, Cambridge University Press, 1999.

[12] S. Gupta, M. K. Markey, J. K. Aggarwal, and A. C. Bovik, "Three dimensional face recognition based on geodesic and Euclidean distances," in Proc. SPIE Vis. Geom., 2007, vol. 6499, pp. 64990D-64990D-11.

[13] J. Petersson, T. Brismar, and Ö. Smedby, "Analysis of skeletal microstructure with clinical multislice CT," in Proc. MICCAI, LNCS 4191, 2006, pp. 880-887. 